\title{
Implementation of MPOWER Package in the South-East Asia Region: Evidence from the WHO Report on the Global Tobacco Epidemic (2009-2021)
}

\author{
Jagdish Kaur ${ }^{1}$, Arvind Vashishta Rinkoo ${ }^{1 *}$, Hebe N Gouda ${ }^{2}$, Vinayak Prasad ${ }^{3}$, \\ Razia N Pendse ${ }^{4}$
}

\begin{abstract}
The WHO MPOWER package is a set of six evidence-based and cost-effective measures which was introduced on 7 February 2008 to facilitate the implementation of the provisions of the WHO Framework Convention on Tobacco Control at the ground level. These measures are: Monitoring tobacco use and prevention policies (M); Protecting people from tobacco smoke (P); Offering help to quit tobacco use (O); Warning about the dangers of tobacco (W); Enforcing bans on tobacco advertising, promotion and sponsorship (E); and Raising taxes on tobacco (R). Since its launch, the MPOWER package has become the guiding principle for all the countries of the South-East Asia Region in their crusade against the tobacco epidemic. This review article tracks the implementation of the MPOWER measures in the 11 member countries of the Region based on the last seven WHO Report on the Global Tobacco Epidemic (GTCR), i.e., GTCR2/2009-GTCR $8 / 2021$. This is with an aim to enable the countries to review their progress in implementing the MPOWER measures and to take steps to improve their advancement towards reducing the demand for tobacco products at the country level.
\end{abstract}

Keywords: Tobacco control- MPOWER package- South-East Asia Region- noncommunicable diseases- tobacco taxation

Asian Pac J Cancer Prev, 22, Progress of Tobacco Control in the South-East Asia Region Suppl, 71-80

Setting the context

In the WHO South-East Asia (SEA) Region, there are about 230 million adult smokers and 248 million adult smokeless tobacco users as per the third edition of the WHO Global Report on trends in prevalence of tobacco smoking (2000-2025). This makes the Region one of the largest consumers of tobacco, housing more than one fifth of all adult tobacco smokers and more than $80 \%$ of all adult smokeless tobacco users globally (WHO, 2019b).

The WHO MPOWER package is a set of six evidence-based and cost-effective measures which was introduced on 7 February 2008 to facilitate the implementation of the provisions of the WHO Framework Convention on Tobacco Control at the ground level. These measures are: Monitoring tobacco use and prevention policies (M); Protecting people from tobacco smoke (P); Offering help to quit tobacco use $(\mathrm{O})$; Warning about the dangers of tobacco (W); Enforcing bans on tobacco advertising, promotion and sponsorship (E); and Raising taxes on tobacco (R). Since its launch, the MPOWER package has become the guiding principle for all the countries of the SEA Region in their crusade against the tobacco epidemic (WHO, 2018).

Countries of the Region have been striving to make incremental progress in the implementation of various provisions of the MPOWER package. Many of these measures are also the recommended "best buys" for effective tobacco control under the Global NCD Action Plan. The WHO Report on the Global Tobacco Epidemic (GTCR) is a biennial report that reviews the status of implementation of MPOWER measures around the world. Till now, eight GTCR reports have been published - from GTCR1 in 2008 to GTCR8 in 2021.

As per the third edition of the WHO Global Report on trends in prevalence of tobacco smoking (2000-2025), the prevalence of tobacco use in the SEA Region appears to be decreasing; however, the Region may just fall short of the target of a $30 \%$ relative reduction in prevalence between 2010 and 2025 . As per the report, a gap of $0.5 \%$ still persists between the 2025 target prevalence $(24.6 \%)$ and the 2025 projected prevalence $(25.1 \%)$ of tobacco use by people aged $>15$ years in the Region (WHO, 2019b).

${ }^{1}$ Tobacco-Free Initiative, Regional Office for the South-East Asia, World Health Organization, India. ${ }^{2}$ Tobacco Free Initiative, Department of Health Promotion, WHO HQ, Switzerland. ${ }^{3}$ Unit Head, No Tobacco, WHO HQ, Switzerland. ${ }^{4}$ Director, Department of Healthier Populations and Non Communicable Diseases (HPN), Regional Office for the South-East Asia, World Health Organization, India.*For Correspondence: docavr@gmail.com 
Thus, if the Region has to attain the global targets, it is imperative for the member countries to further strengthen their tobacco control efforts, including those aimed at more effective implementation of the WHO MPOWER package.

This review article tracks the implementation of the MPOWER measures in the 11 member countries of the SEA Region based on the last seven GTCR reports (GTCR2/2009-GTCR8/2021). This is with an aim to enable the countries to review their progress in implementing the MPOWER measures and to take steps to improve their advancement towards reducing the demand for tobacco products at the country level.

\section{Monitoring tobacco use and prevention policies}

Availability of recent, representative and periodic data on tobacco use for both adults and youth indicates the highest level of achievement in monitoring tobacco use and prevention policies. Currently, Bangladesh, Bhutan, Indonesia, Myanmar and Thailand are the countries of the Region at the best-practice level (WHO, 2021). Out of these, Bangladesh, Bhutan and Myanmar have exceptionally been retained in the best-practice group (based on their performance in GTCR 2019) in GTCR 2021 owing to the challenges of running national populationbased surveys during the COVID-19 pandemic (WHO, 2019a; WHO, 2021). In GTCR 2009, only one country (India) of the Region was in the best-practice group (WHO, 2009). In 2011, this increased to five countries (India, Indonesia, Myanmar, Sri Lanka and Thailand) (WHO, 2011). In 2013 and 2015, only two countries (India \& Thailand and Nepal \& Thailand, respectively) were at the highest level of achievement (WHO, 2013; WHO, 2015). In GTCR 2017, four countries (Bhutan, Indonesia, Myanmar and Thailand) were retained in the best-practice group (WHO, 2017). Notably, Thailand has been the most consistent in its performance. Please refer to Figure 1 and table 1 for details. DPR Korea, Maldives and Timor-Leste are the only countries in the Region that have never been at the highest level of achievement during the period 2009-2021. Additionally, in GTCR 2021, Indonesia and Nepal have been included in the countries that monitor Electronic nicotine delivery systems (ENDS) use in adults. Likewise, Thailand has been included among the countries that monitor ENDS use in adolescents (WHO, 2021).

\section{Protecting people from tobacco smoke}

As per GTCR 2017, 2019 and 2021, Nepal and Thailand are the only two countries in the Region that have all the public places completely smoke-free (WHO, 2017; WHO, 2019a; WHO, 2021). Additionally, as per GTCR 2021, Bhutan and DPR Korea need to cover only one more place (cafés, pubs \& bars and government facilities, respectively) with a smoking ban to join the exclusive list of countries with comprehensive smoke free laws (WHO, 2021). In GTCR 2009, only one country (Bhutan) of the Region was in the best-practice group (WHO, 2009). In 2011, this increased to three countries (Bhutan, Maldives and Thailand) (WHO, 2011). In both 2013 and 2015, the same three countries (Bhutan, Nepal and Thailand) were at the highest level of achievement (WHO, 2013; WHO, 2015). Nepal and Thailand have been the most consistent in this category. Please refer to figure 2 and table 2 for details. India has one or more public places where designated smoking rooms are allowed. Separate, completely enclosed smoking rooms are only allowed if they are separately ventilated to the outside and/or kept under negative air pressure in relation to the surrounding areas. Given the difficulty of meeting the very strict requirements delineated for such rooms, they appear to be a practical impossibility. No reliable empirical evidence is presently available to ascertain whether they have been constructed (WHO, 2019a). Bangladesh, Indonesia, Maldives and Myanmar have only three to five public places completely smoke-free, and need to more holistically formulate and implement smokefree laws (WHO, 2021).

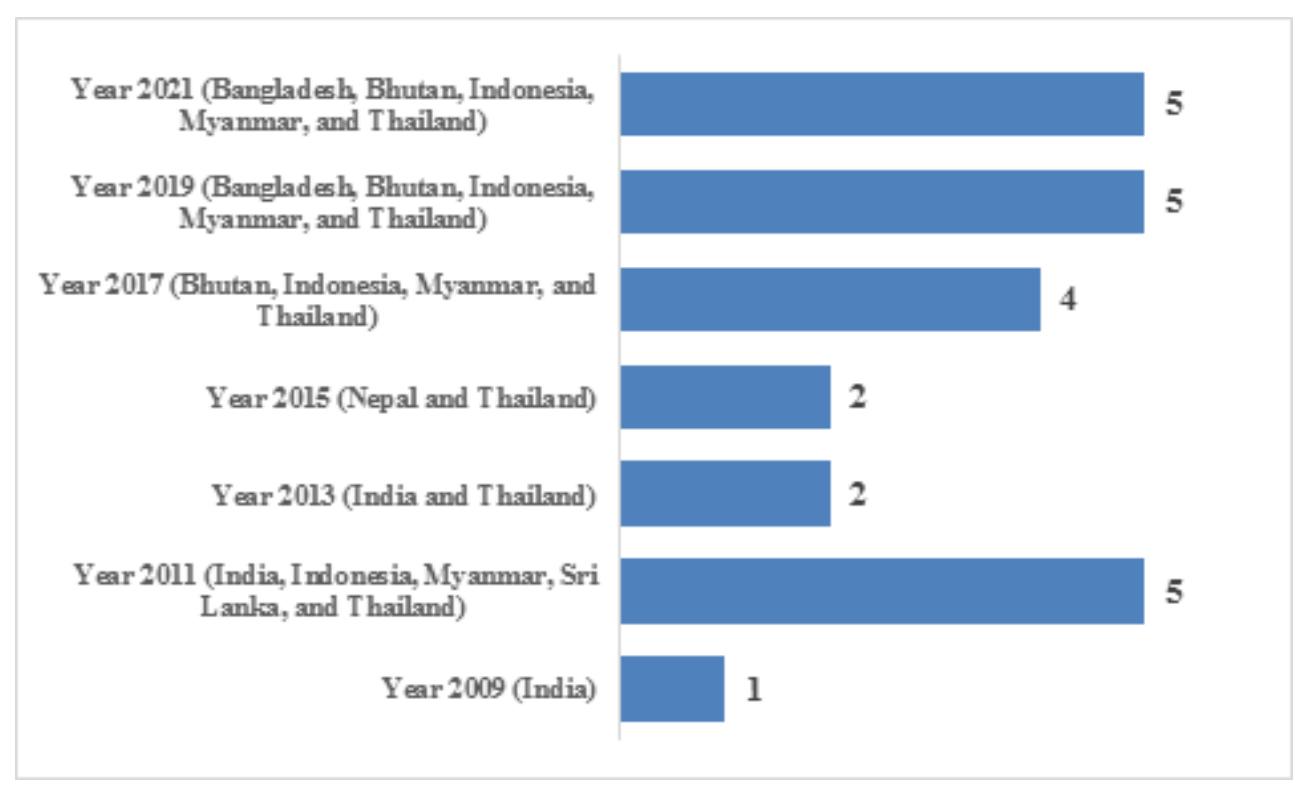

Figure 1. Number of Countries in the South-East Asia Region with Highest Level of Achievement (Availability of Recent, Representative, and Periodic Data on Tobacco Use for Both Adults and Youth) in Monitoring Tobacco Use Over the Period 2009-2021 


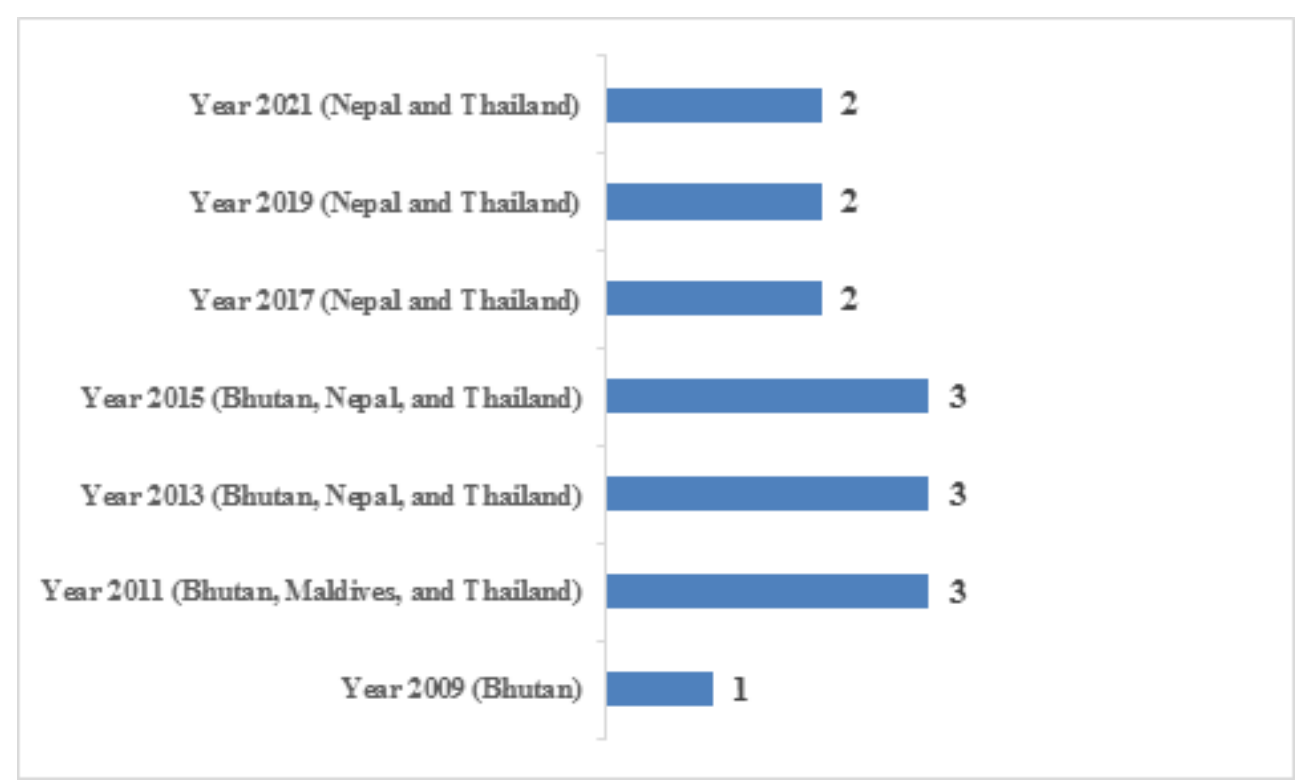

Figure 2. Number of Countries in the South-East Asia Region with Highest Level of Achievement (All Public Places Completely Smokefree) in Policies on Smokefree Environments Over the Period 2009-2021

\section{Offering help to quit tobacco use}

In general, affordable tobacco cessation services are lacking in the Region (WHO, 2020). Exceptionally, India has strategically invested in scaling up tobacco cessation through mCessation services and the toll-free National Tobacco Quitline, which has four hubs servicing different regions of the country. The Quitline is staffed by 100 trained counselors providing services in over 15 languages to address the needs of 267 million adult tobacco users in India (WHO, 2021). In 2017, 2019 and 2021, India has been placed in the best practice group i.e., countries having a national quit line, and both NRT and some cessation services cost-covered (WHO, 2017; WHO, 2019a; WHO, 2021). Additionally, in 2013, Thailand was placed in this coveted list (WHO, 2013). Apart from India and Thailand, no other country in the Region has ever been included in the best-practice category over the years 2009-2021. Please refer to figure 3 and table 3 for details.

Prioritizing availability and implementation of efficient and affordable tobacco cessation services ought to be the way forward for the Region. Tobacco cessation services, particularly those aimed at youth at the population level, need to be strengthened across the countries. Nicotine replacement therapy should be made available in the public health system. A national tobacco cessation strategy ought to be in place in all the countries. A national toll-free quitline number should be included in all tobacco control campaigns. Emerging technologies such as mTobaccoCessation should be leveraged. Quality counselling services and brief advice should be made available in school settings by training school health counselors and integrating the services with primary health

Table 1. Implementation of Measures Related to Monitoring Tobacco Uuse and Prevention Policies (M) in the Countries of the SEA Region Over the Period 2009-2021

\begin{tabular}{|c|c|c|c|c|c|c|c|}
\hline Country & GTCR 2009 & GTCR 2011 & GTCR 2013 & GTCR 2015 & GTCR 2017 & GTCR 2019 & GTCR 2021 \\
\hline \multicolumn{8}{|l|}{ Bangladesh } \\
\hline \multicolumn{8}{|l|}{ Bhutan } \\
\hline \multicolumn{8}{|l|}{ DPR Korea } \\
\hline \multicolumn{8}{|l|}{ India } \\
\hline \multicolumn{8}{|l|}{ Indonesia } \\
\hline \multicolumn{8}{|l|}{ Maldives } \\
\hline \multicolumn{8}{|l|}{ Myanmar } \\
\hline \multicolumn{8}{|l|}{ Nepal } \\
\hline \multicolumn{8}{|l|}{ Sri Lanka } \\
\hline \multicolumn{8}{|l|}{ Thailand } \\
\hline \multicolumn{8}{|l|}{ Timor-Leste } \\
\hline & \multicolumn{7}{|c|}{ No known data or no recent data or data that are not both recent and representative } \\
\hline & \multicolumn{7}{|c|}{ Recent and representative data for either adults or youth } \\
\hline & \multicolumn{7}{|c|}{ Recent and representative data for both adults and youth } \\
\hline & \multicolumn{7}{|c|}{ Recent, representative and periodic data for both adults and youth } \\
\hline
\end{tabular}

GTCR, WHO Report on the Global Tobacco Epidemic 


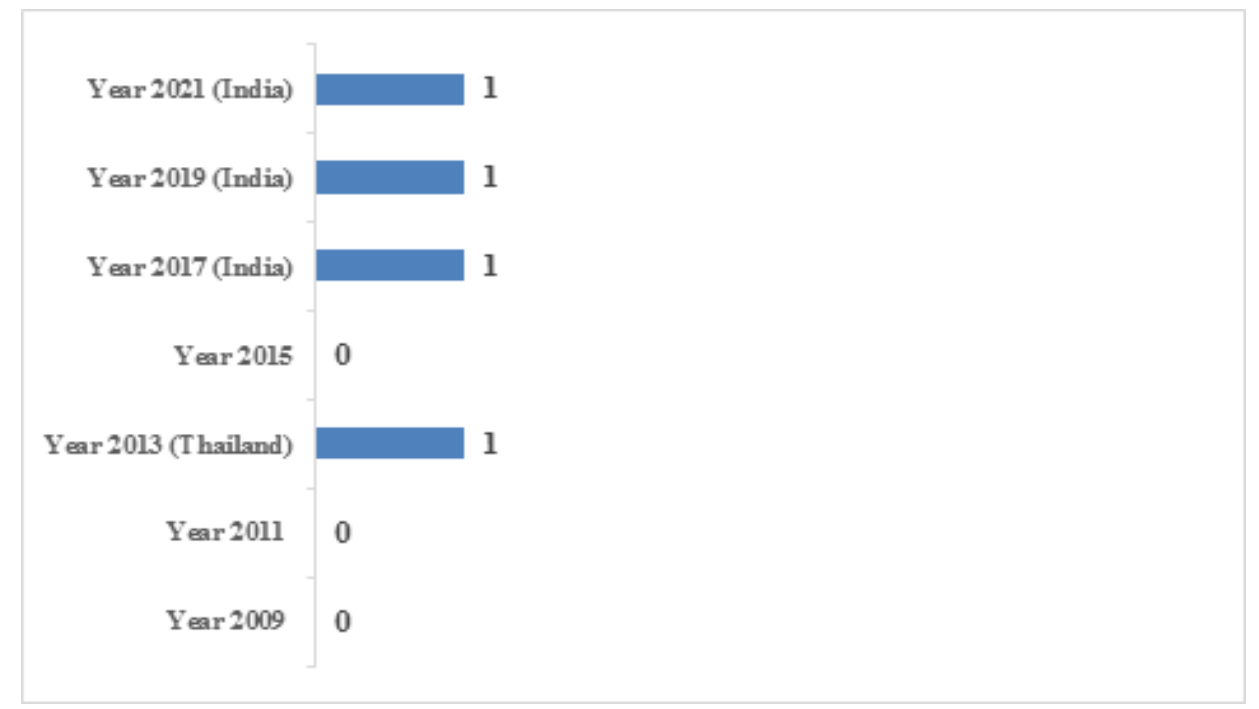

Figure 3. Number of Countries in the South-East Asia Region with Highest Level of Achievement (National Quitline, and both NRT and some Cessation Services Cost-Covered) in Treatment of Tobacco Dependence over the Period 2009-2021

care and the implementation of the WHO PEN package.

\section{Warning about the dangers of tobacco}

The Region is performing progressively well in implementation of large graphic health warnings and is, in fact, one of the best performing WHO regions in doing so. Please refer to Figure 4 and Table 4 for details. With only one country (Thailand) in the best practice group (large warnings with all appropriate characteristics) in 2011, the Region has incrementally progressed and has as many as six countries (Bangladesh, India, Nepal, Sri Lanka, Thailand and Timor-Leste) in the elite group in 2021 (WHO, 2011; WHO, 2021). Besides, Thailand is one among the 17 countries of the world which had adopted legislation mandating plain packaging of tobacco products and had issued regulations with implementation dates (WHO, 2021). Maldives recently improved its legislation paving way for implementation of large and effective graphic health warnings. Myanmar is also implementing large graphic health warnings (WHO, 2018). Bhutan and DPR Korea have consistently lagged behind in implementing effective health warnings on packages of tobacco products and need to catch up. In mass media campaigns, Indonesia, Myanmar, Thailand and Timor-Leste are currently at the best practice level by conducting regular national anti-tobacco campaigns with at least seven appropriate characteristics including airing on television and/or radio (WHO, 2021). Please refer to

Table 2. Implementation of Measures Related to Protecting People from Tobacco Smoke (P) in the Countries of the SEA Region Over the Period 2009-2021

\begin{tabular}{|c|c|c|c|c|c|c|c|}
\hline Country & GTCR 2009 & GTCR 2011 & GTCR 2013 & GTCR 2015 & GTCR 2017 & GTCR 2019 & GTCR 2021 \\
\hline \multicolumn{8}{|l|}{ Bangladesh } \\
\hline \multicolumn{8}{|l|}{ Bhutan } \\
\hline \multicolumn{8}{|l|}{ DPR Korea } \\
\hline \multicolumn{8}{|l|}{ India } \\
\hline \multicolumn{8}{|l|}{ Indonesia } \\
\hline \multicolumn{8}{|l|}{ Maldives } \\
\hline \multicolumn{8}{|l|}{ Myanmar } \\
\hline \multicolumn{8}{|l|}{ Nepal } \\
\hline \multicolumn{8}{|l|}{ Sri Lanka } \\
\hline \multicolumn{8}{|l|}{ Thailand } \\
\hline \multicolumn{8}{|l|}{ Timor-Leste } \\
\hline & \multicolumn{7}{|c|}{ Data not reported } \\
\hline & \multicolumn{7}{|c|}{ Complete absence of ban, or up to two public places completely smoke-free } \\
\hline & \multicolumn{7}{|c|}{ Three to five public places completely smoke-free } \\
\hline & \multicolumn{7}{|c|}{ Six to seven public places completely smoke-free } \\
\hline & \multicolumn{7}{|c|}{$\begin{array}{l}\text { All public places completely smoke-free (or at least } 90 \% \text { of the population covered by complete subnational } \\
\text { smoke-free legislation) }\end{array}$} \\
\hline
\end{tabular}

GTCR, WHO Report on the Global Tobacco Epidemic 


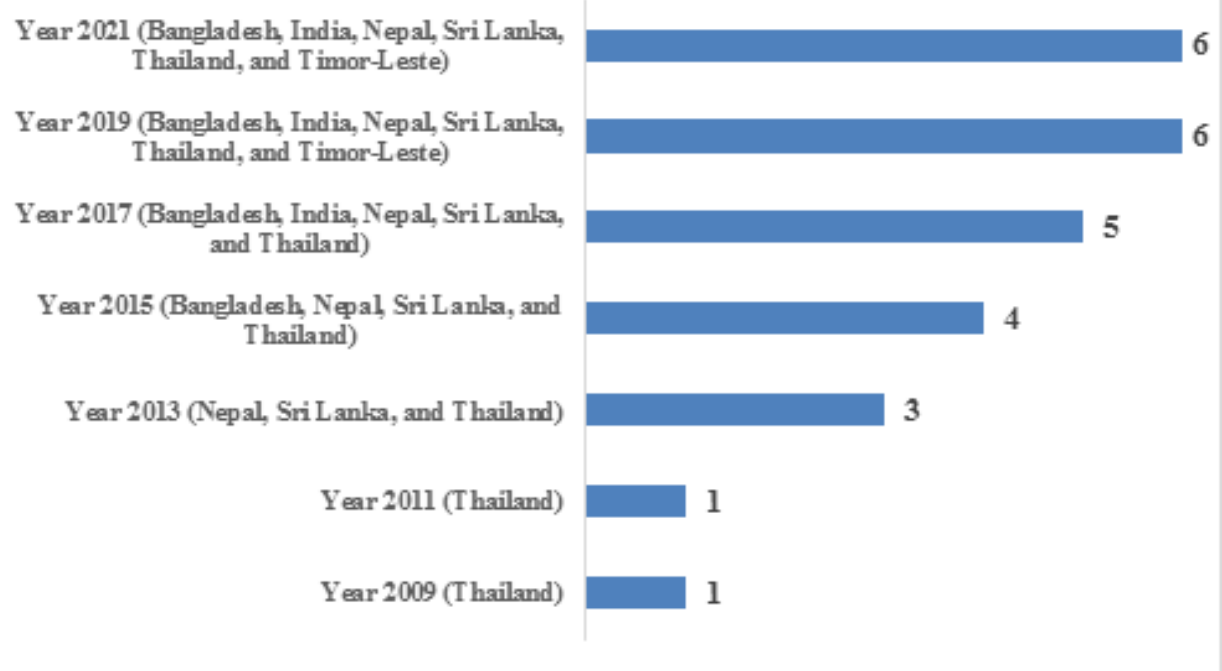

Figure 4. Number of Countries in the South-East Asia Region with Highest Level of Achievement (Large Warnings with All Appropriate Characteristics) in Health Warnings on Cigarette Packages Over the Period 2009-2021

Figure 5 and Table 5 for details. Maldives and Sri Lanka have been inconsistent and need to regularly conduct and sustain effective tobacco control mass media campaigns (WHO, 2018; WHO, 2019a; WHO, 2021).

Enforcing bans on tobacco advertising, promotion and sponsorship

Maldives and Nepal have comprehensive bans on all forms of tobacco advertising, promotion and sponsorship (TAPS). Since 2015, these two countries have consistently been in the best-practice group (WHO, 2015; WHO, 2017; WHO, 2019a; WHO, 2021). In 2009, four countries (DPR Korea, Myanmar, Sri Lanka and Thailand) were in the best-practice group (WHO, 2009). In 2011 and 2013, this number decreased to two (Myanmar and Thailand) and one (Maldives), respectively (WHO, 2011; WHO, 2013). Please refer to figure 6 and table 6 for details. As of now, India needs only to ban advertising of tobacco products at point of sale to achieve the highest level in ban on TAPS. Likewise, Sri Lanka and Thailand need only to ban brand-stretching to achieve the highest level (WHO, 2021). In particular, DPR Korea and Indonesia need to prioritize comprehensive TAPS ban for effectively tackling the tobacco epidemic (WHO, 2013; WHO, 2015; WHO, 2017; WHO, 2018; WHO, 2019a; WHO, 2021).

\section{Raising taxes on tobacco}

It is recommended to raise taxes to at least $75 \%$ of the retail price of the most widely sold brand of cigarettes. However, the countries of the Region have

Table 3. Implementation of Measures Related to Offering Help to Quit Tobacco Use $(\mathrm{O})$ in the Countries of the SEA Region Over the Period 2009-2021

\begin{tabular}{|c|c|c|c|c|c|c|c|}
\hline Country & GTCR 2009 & GTCR 2011 & GTCR 2013 & GTCR 2015 & GTCR 2017 & GTCR 2019 & GTCR 2021 \\
\hline \multicolumn{8}{|l|}{ Bangladesh } \\
\hline \multicolumn{8}{|l|}{ Bhutan } \\
\hline \multicolumn{8}{|l|}{ DPR Korea } \\
\hline \multicolumn{8}{|l|}{ India } \\
\hline \multicolumn{8}{|l|}{ Indonesia } \\
\hline \multicolumn{8}{|l|}{ Maldives } \\
\hline \multicolumn{8}{|l|}{ Myanmar } \\
\hline \multicolumn{8}{|l|}{ Nepal } \\
\hline \multicolumn{8}{|l|}{ Sri Lanka } \\
\hline \multicolumn{8}{|l|}{ Thailand } \\
\hline \multicolumn{8}{|l|}{ Timor-Leste } \\
\hline & \multicolumn{7}{|c|}{ Data not reported } \\
\hline & \multicolumn{7}{|l|}{ None } \\
\hline & \multicolumn{7}{|c|}{ NRT and/or some cessation services (neither cost-covered) } \\
\hline & \multicolumn{7}{|c|}{ NRT and/or some cessation services (at least one of which is cost-covered) } \\
\hline & \multicolumn{7}{|c|}{ National quit line, and both NRT and some cessation services cost-covered } \\
\hline
\end{tabular}

GTCR, WHO Report on the Global Tobacco Epidemic 


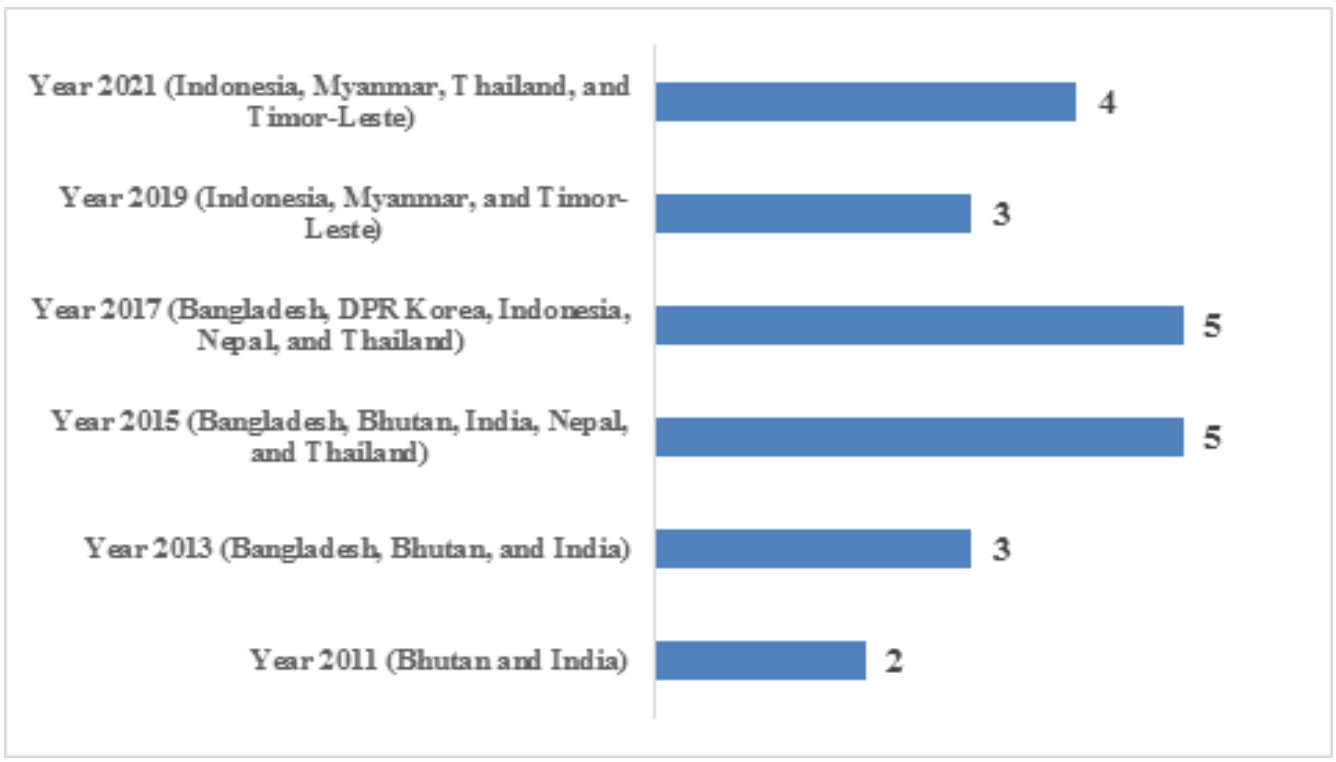

Figure 5. Number of Countries in the South-East Asia Region with Highest Level of Achievement (National Campaign Conducted with at Least Seven Appropriate Characteristics Including Airing on Television and/or Radio) in Anti-Tobacco Mass Media Campaigns Over the Period 2011-2021

been inconsistent in their efforts to raise tobacco taxes to the optimal level. It is imperative for the countries of the Region to raise taxes on all tobacco products, including smokeless tobacco products and indigenous smoking products, to decrease the demand for these products. In GTCR 2021, only Sri Lanka and Thailand have been placed in the best-practice group by levying taxes that comprise at least $75 \%$ of retail prices $(77.02 \%$ in case of Sri Lanka and $78.6 \%$ in casFe of Thailand). In fact, globally, the most significant tax share increase during the period 2018-2020 was made by Sri Lanka, whose 2018 rate of $66.17 \%$ was raised to $77.02 \%$ by 2020 (WHO, 2021). In 2015 and 2017, only Bangladesh was in the elite group (WHO, 2015; WHO, 2017). In 2009, 2011 and 2013, none of the countries in the Region could make it to the best-practice group (WHO, 2009; WHO, 2011; WHO, 2013). In 2019, Thailand was the only country in the Region that was in the best-practice category (WHO, 2019). Please refer to figure 7 and table 7 for details. Additionally, the Region is trying to leverage tobacco taxation revenue for funding tobacco control in the spirit of the Addis Ababa Action Agenda (Thai Health Promotion

Table 4. Implementation of Measures Related to Health Warnings on Cigarette Packages (W) in the Countries of the SEA Region Over the Period 2009-2021

\begin{tabular}{|c|c|c|c|c|c|c|c|}
\hline Country & GTCR 2009 & GTCR 2011 & GTCR 2013 & GTCR 2015 & GTCR 2017 & GTCR 2019 & GTCR 2021 \\
\hline \multicolumn{8}{|c|}{ Bangladesh } \\
\hline \multicolumn{8}{|l|}{ Bhutan } \\
\hline \multicolumn{8}{|c|}{ DPR Korea } \\
\hline \multicolumn{8}{|l|}{ India } \\
\hline \multicolumn{8}{|c|}{ Indonesia } \\
\hline \multicolumn{8}{|c|}{ Maldives } \\
\hline \multicolumn{8}{|c|}{ Myanmar } \\
\hline \multicolumn{8}{|l|}{ Nepal } \\
\hline \multicolumn{8}{|c|}{ Sri Lanka } \\
\hline \multicolumn{8}{|l|}{ Thailand } \\
\hline \multicolumn{8}{|c|}{ Timor-Leste } \\
\hline & \multicolumn{7}{|c|}{ Data not reported } \\
\hline & \multicolumn{7}{|c|}{ No warnings or small warnings } \\
\hline & \multicolumn{7}{|c|}{$\begin{array}{l}\text { Medium size warnings missing some appropriate characteristics OR large warnings missing many appro- } \\
\text { priate characteristics }\end{array}$} \\
\hline & \multicolumn{7}{|c|}{$\begin{array}{l}\text { Medium size warnings with all appropriate characteristics OR large warnings missing some appropriate } \\
\text { characteristics }\end{array}$} \\
\hline & \multicolumn{7}{|c|}{ Large warnings with all appropriate characteristics } \\
\hline
\end{tabular}

GTCR, WHO Report on the Global Tobacco Epidemic 

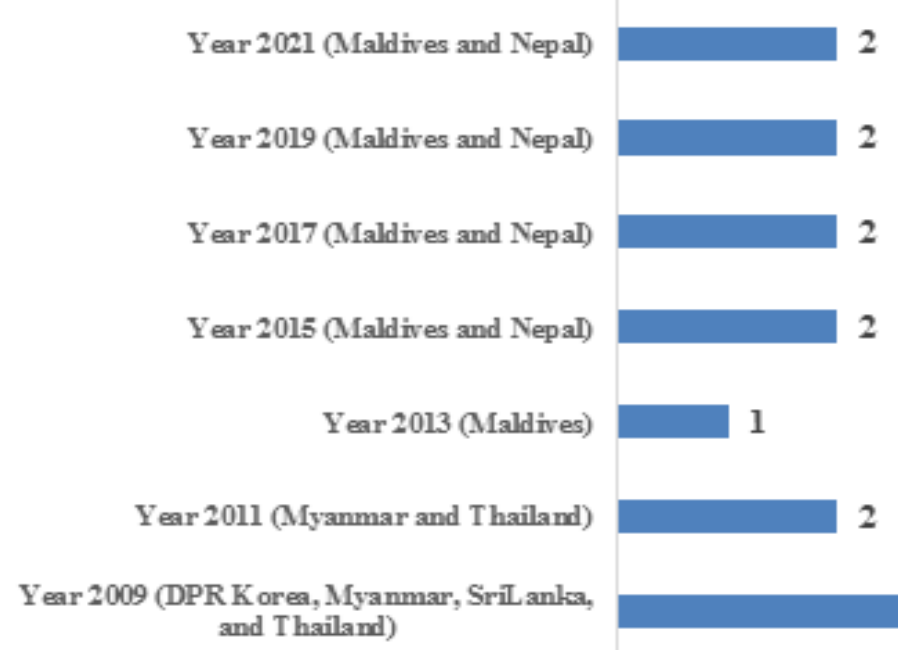

2

2
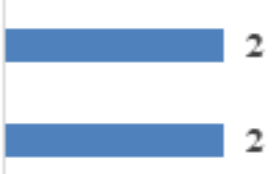

2
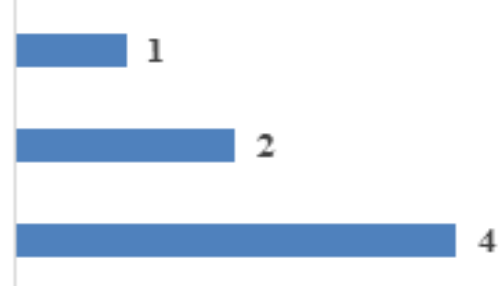

Figure 6. Number of Countries in the South-East Asia Region with Highest Level of Achievement (Ban on All Forms of Direct and Indirect Advertising) in Bans on Tobacco Advertisement, Promotion, and Sponsorship Over the Period 2009-2021

Foundation in Thailand, guidelines for utilizing local tobacco tax revenue for health in Indonesia, and 1\% health development surcharge for financing health programmes in Bangladesh).

\section{Way forward}

It is encouraging to note that the countries of the Region are progressively improving the implementation of MPOWER package in their jurisdictions. However, the momentum needs to be carried forward. DPR Korea, Maldives and Timor-Leste need to improve monitoring of tobacco use and prevention policies. Bangladesh, Indonesia, Maldives and Myanmar need to more holistically formulate and implement smokefree laws. The Region should prioritize availability and implementation of efficient and affordable quality tobacco cessation services, particularly those aimed at youth at the population level. Bhutan and DPR Korea have consistently lagged behind in implementing effective health warnings on packages of tobacco products. Bhutan, Maldives, Nepal and Sri Lanka ought to regularly conduct and sustain effective tobacco control mass media campaigns. DPR Korea and Indonesia should prioritize comprehensive TAPS ban for effectively tackling the tobacco epidemic. It is imperative for the countries of the Region to raise taxes, to at least $75 \%$ of the retail price of the most widely

Table 5. Implementation of Measures Related to Mass Media Anti-Tobacco Campaigns (W) in the Countries of the SEA Region Over the Period 2011-2021

\begin{tabular}{|c|c|c|c|c|c|c|}
\hline Country & GTCR 2011 & GTCR 2013 & GTCR 2015 & GTCR 2017 & GTCR 2019 & GTCR 2021 \\
\hline \multicolumn{7}{|c|}{ Bangladesh } \\
\hline \multicolumn{7}{|l|}{ Bhutan } \\
\hline \multicolumn{7}{|c|}{ DPR Korea } \\
\hline \multicolumn{7}{|l|}{ India } \\
\hline \multicolumn{7}{|l|}{ Indonesia } \\
\hline \multicolumn{7}{|l|}{ Maldives } \\
\hline \multicolumn{7}{|l|}{ Myanmar } \\
\hline \multicolumn{7}{|l|}{ Nepal } \\
\hline \multicolumn{7}{|l|}{ Sri Lanka } \\
\hline \multicolumn{7}{|l|}{ Thailand } \\
\hline \multicolumn{7}{|c|}{ Timor-Leste } \\
\hline & \multicolumn{6}{|l|}{ Data not reported } \\
\hline & \multicolumn{6}{|c|}{ No national campaign conducted during the reporting period with a duration of at least 3 weeks } \\
\hline & \multicolumn{6}{|c|}{ National campaign conducted with one to four appropriate characteristics } \\
\hline & \multicolumn{6}{|c|}{ National campaign conducted with five to six appropriate characteristics } \\
\hline & \multicolumn{6}{|c|}{$\begin{array}{l}\text { National campaign conducted with at least seven appropriate characteristics including airing on television } \\
\text { and/or radio }\end{array}$} \\
\hline
\end{tabular}

GTCR, WHO Report on the Global Tobacco Epidemic 


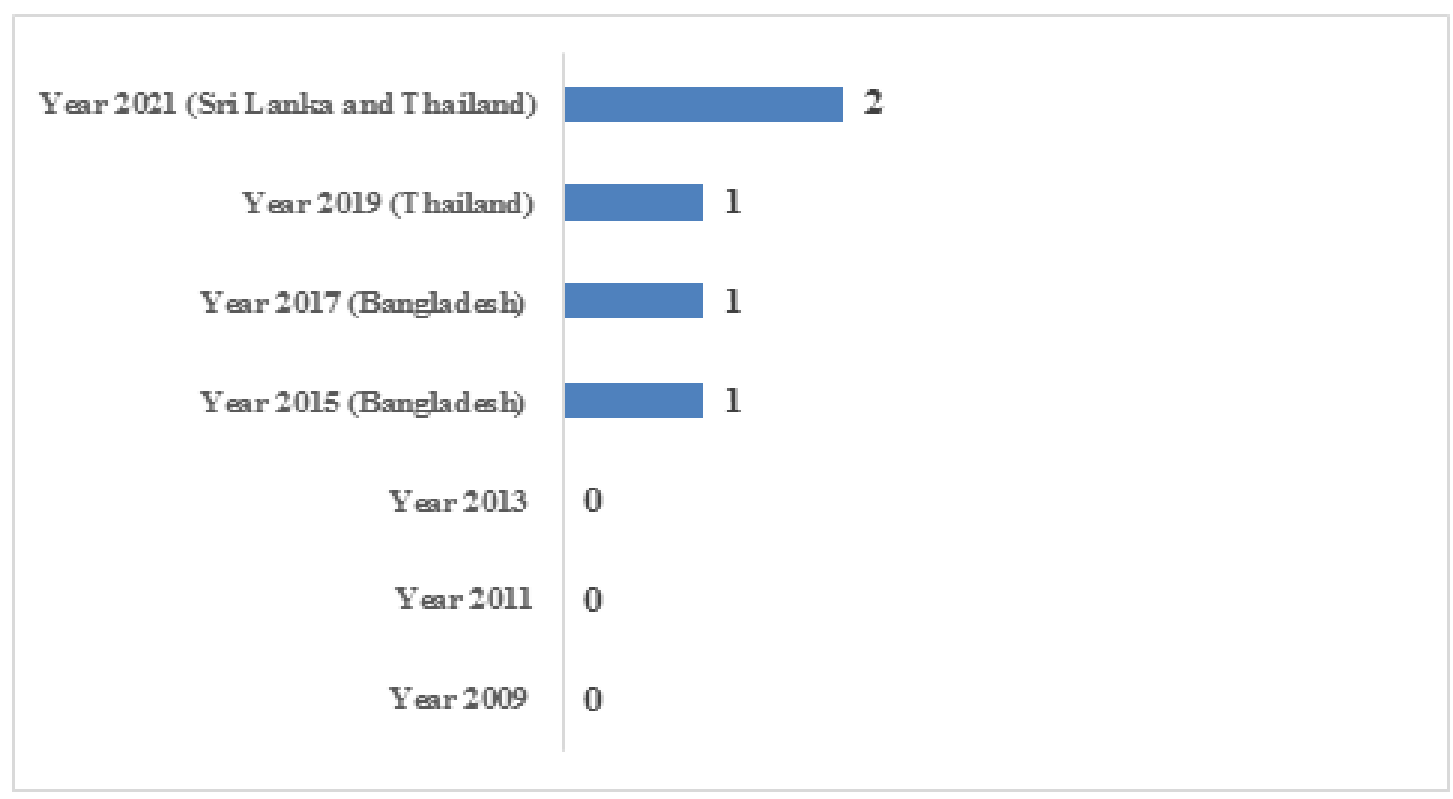

Figure 7. Number of Countries in the South-East Asia Region with at Least 75\% Share of Tobacco-Specific Taxes in Retail Price of the most Widely Sold Brand of Cigarettes over the Period 2009-2021

sold brand, on all tobacco products, including smokeless tobacco products and indigenous smoking products such as bidi, cheroots or kreteks, to realistically decrease the demand for these products. The countries of the Region should look at taxing all forms of tobacco equitably.

Despite making significant policy progress, the countries of the Region are falling short of the target of a $30 \%$ relative reduction in prevalence of tobacco use (between the years 2010 and 2025) by people aged $>15$ years (WHO, 2019b). Thus, effective implementation of tobacco control regulations and policies needs to be prioritized across the Region. Enhanced institutional capacity, operational effectiveness and political commitment at the country level could go a long way in improving enforcement of extant tobacco control laws. Dedicated well-structured National Tobacco Control Programmes with earmarked budgets and resources (as in India) can help transform policy progress into actual public health gains at the population level. Besides, lately, the dynamics of tobacco epidemic in the SEA Region seems to be changing. The tobacco industry is increasingly targeting the implementation of tobacco control measures. New tobacco products such as ENDS and heated tobacco products (HTPs) have emerged aimed at renormalizing

Table 6. Implementation of Measures Related to Enforcing Bans on Tobacco Advertising, Promotion and Sponsorship (E) in the Countries of the SEA Region over the Period 2009-2021

\begin{tabular}{|c|c|c|c|c|c|c|c|}
\hline Country & GTCR 2009 & GTCR 2011 & GTCR 2013 & GTCR 2015 & GTCR 2017 & GTCR 2019 & GTCR 2021 \\
\hline \multicolumn{8}{|l|}{ Bangladesh } \\
\hline \multicolumn{8}{|l|}{ Bhutan } \\
\hline \multicolumn{8}{|l|}{ DPR Korea } \\
\hline \multicolumn{8}{|l|}{ India } \\
\hline \multicolumn{8}{|l|}{ Indonesia } \\
\hline \multicolumn{8}{|l|}{ Maldives } \\
\hline \multicolumn{8}{|l|}{ Myanmar } \\
\hline \multicolumn{8}{|l|}{ Nepal } \\
\hline \multicolumn{8}{|l|}{ Sri Lanka } \\
\hline \multicolumn{8}{|l|}{ Thailand } \\
\hline \multicolumn{8}{|l|}{ Timor-Leste } \\
\hline & \multicolumn{7}{|c|}{ Data not reported } \\
\hline & \multicolumn{7}{|c|}{ Complete absence of ban, or ban that does not cover national television, radio and print media } \\
\hline & \multicolumn{7}{|c|}{ Ban on national television, radio and print media only } \\
\hline & \multicolumn{7}{|c|}{$\begin{array}{l}\text { Ban on national television, radio and print media as well as on some but not all other forms of direct and/or } \\
\text { indirect advertising }\end{array}$} \\
\hline & \multicolumn{7}{|c|}{$\begin{array}{l}\text { Ban on all forms of direct and indirect advertising (or at least } 90 \% \text { of the population covered by subnational } \\
\text { legislation completely banning tobacco advertising, promotion and sponsorship) }\end{array}$} \\
\hline
\end{tabular}

GTCR, WHO Report on the Global Tobacco Epidemic 
Table 7. Implementation of Measures Related to Raising Tax on Tobacco (R) in Terms of Share of Total Taxes in the Retail Price of the most Widely Sold Brand of Cigarettes in the Countries of the SEA Region over the Period 2009-2021

\begin{tabular}{|c|c|c|c|c|c|c|c|}
\hline Country & GTCR 2009 & GTCR 2011 & GTCR 2013 & GTCR 2015 & GTCR 2017 & GTCR 2019 & GTCR 2021 \\
\hline \multicolumn{8}{|l|}{ Bangladesh } \\
\hline \multicolumn{8}{|l|}{ Bhutan } \\
\hline \multicolumn{8}{|l|}{ DPR Korea } \\
\hline \multicolumn{8}{|l|}{ India } \\
\hline \multicolumn{8}{|l|}{ Indonesia } \\
\hline \multicolumn{8}{|l|}{ Maldives } \\
\hline \multicolumn{8}{|l|}{ Myanmar } \\
\hline \multicolumn{8}{|l|}{ Nepal } \\
\hline \multicolumn{8}{|l|}{ Sri Lanka } \\
\hline \multicolumn{8}{|l|}{ Thailand } \\
\hline \multicolumn{8}{|l|}{ Timor-Leste } \\
\hline & \multicolumn{7}{|c|}{ Data not reported/Data not required/Not applicable/Not available } \\
\hline & \multicolumn{7}{|c|}{$<25 \%$ of retail price is tax } \\
\hline & \multicolumn{7}{|c|}{$\geq 25 \%$ and $<50 \%$ of retail price is tax } \\
\hline & \multicolumn{7}{|c|}{$\geq 50 \%$ and $<75 \%$ of retail price is tax } \\
\hline & \multicolumn{7}{|c|}{$\geq 75 \%$ of retail price is tax } \\
\hline
\end{tabular}

GTCR, WHO Report on the Global Tobacco Epidemic

tobacco use, especially among youth and teenagers (Kaur and Rinkoo, 2017). The smokeless tobacco and areca nut are emerging as major stumbling blocks (Kaur, Thamarangsi and Rinkoo, 2017). Thus, it is important for the countries of the Region to be proactively vigilant about these emerging challenges.

Also, supply side issues - addressing illicit trade in tobacco products, restricting access of tobacco products to minors and provisioning alternative livelihoods for tobacco growers and workers - are important for the Region. Unfortunately, as of now, only India and Sri Lanka are Parties to the Protocol to Eliminate Illicit Trade in Tobacco Products (WHO, 2020). Countries such as Bhutan, Maldives, Myanmar and Timor-Leste with porous international borders should immediately become Parties to the Protocol. Also, all the countries of the Region should effectively implement provisions related to restricting access of tobacco products to minors. Likewise, a lot of effort has gone into creating evidence on economically viable alternate crops to tobacco over a period of time. Various projects and programmes for alternative livelihoods to tobacco growing have been initiated in the Region, including in Bangladesh, India, Indonesia and Sri Lanka (Kaur, Rinkoo and Arora, 2014; WHO, 2020). However, the initiatives on provisioning viable alternative crop options for tobacco growers in these countries are generally limited to sporadic experiments carried out in research settings. A detailed plan of action at the field level needs to be drawn based on the recommendations of available research.

\section{Author Contribution Statement}

The authors confirm contribution to the paper as follows: Study was conceived by JK. First draft was prepared by AVR and improved by JK. Special inputs were added by HNG, VP, and RNP. All authors reviewed and approved the final version of the manuscript.

\section{Acknowledgements}

The publishing cost was provided by $\mathrm{WHO}$ office in South-East Asia.

\section{Disclaimer}

The opinions or views expressed in this article are solely those of the author(s) and do not express the views or opinions of the organization to which the authors are affiliated.

\section{References}

Kaur J, Rinkoo AV, Arora S (2014). Operationalizing evidence into action for providing viable crop diversification options to tobacco farmers in India-a compelling case for change. Int J Interdisciplinary Multidisciplinary Stud, 2, 148-56

Kaur J, Rinkoo AV (2017). Getting real with the upcoming challenge of electronic nicotine delivery systems: The way forward for the South-East Asia region. Indian J Public Health, 61, 7-11

Kaur J, Thamarangsi T, Rinkoo AV (2017). Regulating smokeless tobacco and processed areca nut in South-East Asia region: The journey so far and the road ahead. Indian J Public Health, 61, 3-6

World Health Organization (2009). WHO report on the global tobacco epidemic 2009: implementing smoke-free environments. Geneva: World Health Organization. Available from: https://apps.who.int/tobacco/ mpower/2009/en/index.html (last accessed 1 July 2021).

World Health Organization (2011). WHO report on the global tobacco epidemic 2011: warning about the dangers of 
tobacco. Geneva: World Health Organization. Available from: https://apps.who.int/tobacco/global_report/2011/en/ index.html (last accessed 5 July 2021).

World Health Organization (2013). WHO report on the global tobacco epidemic 2013: enforcing bans on tobacco advertising, promotion and sponsorship. Geneva: World Health Organization. Available from: https://apps.who.int/ tobacco/global_report/2013/en/index.html (last accessed 8 July 2021).

World Health Organization (2015). WHO report on the global tobacco epidemic 2015: raising taxes on tobacco. Geneva: World Health Organization. Available from: https://apps. who.int/tobacco/global_report/2015/en/index.html (last accessed 11 July 2021).

World Health Organization (2017). WHO report on the global tobacco epidemic 2017: monitoring tobacco use and prevention policies. Geneva: World Health Organization. Available from: https://apps.who.int/tobacco/global_ report/2017/en/index.html (last accessed 13 July 2021).

World Health Organization (2018). Implementation of WHO MPOWER package in the South-East Asia Region: a progress report. World Health Organization. Regional Office for South-East Asia. Available from: https://apps.who.int/ iris/handle/10665/274278 (last accessed 6 August 2021).

World Health Organization (2019a). WHO report on the global tobacco epidemic 2019: offer help to quit tobacco use. Geneva: World Health Organization. Available from: https:// www.who.int/publications/i/item/9789241516204 (last accessed 14 July 2021).

World Health Organization (2019b). WHO global report on trends in prevalence of tobacco use 2000-2025, third edition. Geneva: World Health Organization. Available from: https:// www.who.int/publications/i/item/who-global-report-ontrends-in-prevalence-of-tobacco-use-2000-2025-thirdedition (last accessed 7 July 2021).

World Health Organization (2020). The Tobacco AtlasPerspectives from the South-East Asia Region. World Health Organization, Regional office for South-East Asia. Available from: https://www.who.int/southeastasia/publications-detail/ sear-tobacco-atlas (last accessed 21 July 2021).

World Health Organization (2021). WHO report on the global tobacco epidemic 2021: addressing new and emerging products. Geneva: World Health Organization. Available from: https://www.who.int/publications/i/ item/9789240032095 (last accessed 15 July 2021).

This work is licensed under a Creative Commons AttributionNon Commercial 4.0 International License. 